\title{
Dual short wavelength infrared transillumination/ reflectance mode imaging for caries detection
}

\author{
Yihua Zhu, Marwa Abdelaziz $\odot$, Jacob Simon, Oanh Le, and Daniel Fried $\odot *$ \\ University of California, San Francisco, San Francisco, California, United States
}

\begin{abstract}
Significance: We have developed a clinical probe capable of acquiring near-simultaneous shortwavelength infrared (SWIR) reflectance and occlusal transillumination images of lesions on tooth proximal and occlusal surfaces. We hypothesize that dual images will aid in differentiating between shallow and deep occlusal lesions and reduce the potential of false positives (FPs).
\end{abstract}

Aim: The aim of this study was to test the performance of the dual reflectance and occlusal transillumination probe on extracted teeth prior to commencing clinical studies.

Approach: The dual probe was 3D printed and the imaging system uses an InGaAs camera and broadband superluminescent diode light sources that emit broadband light at $1300 \mathrm{~nm}$ for occlusal transillumination and 1600-nm light for cross-polarization reflectance. The diagnostic performance of the dual probe was assessed using 120 extracted teeth with approximal and occlusal lesions. Reflectance and transillumination images were fused into single images to enhance the contrast between sound and lesion areas. The lesion contrast in both modes did not increase significantly with either the lesion depth or the distance from the occlusal surface for approximal lesions. In addition, the diagnostic performance of radiography, the individual reflectance and transillumination images, dual images, and fused images were compared using micro-computed tomography as the gold standard.

Results: Reflectance imaging at $1600 \mathrm{~nm}$ yielded the highest diagnostic accuracy for lesions on both occlusal and proximal surfaces while radiography yielded the lowest number of FPs.

Conclusion: This study demonstrates that simultaneous acquisition of both reflectance and transillumination SWIR images is possible with a single clinical device.

(C) The Authors. Published by SPIE under a Creative Commons Attribution 4.0 Unported License. Distribution or reproduction of this work in whole or in part requires full attribution of the original publication, including its DOI. [DOI: 10.1117/1.JBO.26.4.043004]

Keywords: short wavelength infrared imaging; caries detection; reflectance; transillumination.

Paper 200352SSR received Nov. 3, 2020; accepted for publication Jan. 5, 2021; published online Jan. 29, 2021.

\section{Introduction}

Short-wavelength infrared (SWIR) and near-IR imaging (NIR) methods have been under development for almost 20 years for use in dentistry and several NIR clinical devices are now available commercially. Due to the high transparency of enamel at longer wavelengths, novel imaging configurations are feasible in which the tooth can be imaged from the occlusal surface after shining light at and below the gum line, which we call occlusal transillumination. ${ }^{1,2}$ Approximal lesions, the lesions located at the proximal contact points in between teeth, can be imaged via occlusal transillumination of the proximal contact points between teeth and by directing SWIR light below the crown while imaging the occlusal surface. ${ }^{2-4}$ The latter approach is capable of imaging occlusal lesions as well with high contrast. ${ }^{1,2,5-8}$ In 2010 , it was demonstrated that approximal lesions that appeared on radiographs could be detected in vivo with SWIR imaging at $1310 \mathrm{~nm}$ with similar sensitivity ${ }^{2}$ and that occlusal transillumination could be employed clinically. This was the first step in demonstrating the clinical potential

*Address all correspondence to Daniel Fried, daniel.fried@ucsf.edu 
of NIR and SWIR imaging for approximal caries detection. In the most recent clinical study in $2016,{ }^{9}$ SWIR transillumination and SWIR reflectance imaging probes were used to screen premolar teeth scheduled for extraction for caries lesions at wavelengths $>1300 \mathrm{~nm}$. The teeth were collected and sectioned into $200-\mu \mathrm{m}$ slices and examined with polarized light microscopy (PLM) and transverse microradiography that served as the gold standard. In addition, extra-oral radiographs were taken of teeth and the diagnostic performance of SWIR imaging was compared with radiography. The sensitivity of the combined SWIR imaging probes was significantly higher $(P<0.05)$ than radiographs for both occlusal and approximal lesions in vivo. It was anticipated that SWIR methods would be more sensitive than radiographs for occlusal lesions since the radiographic sensitivity for occlusal lesions is extremely poor; however, the sensitivity was also much higher for approximal lesions than radiography, 0.53 versus 0.23 . In addition, the sensitivity of each individual SWIR method was either individually equal to or higher than radiography.

Several shorter wavelength NIR devices that utilize either reflectance and transillumination imaging have been introduced commercially operating at 830 and $780 \mathrm{~nm} \cdot{ }^{10-14}$ The shorter wavelength allows the use of less expensive, silicon-based detectors. However, longer wavelength SWIR light has significant advantages. The use of shorter wavelength 830-nm NIR light was first investigated almost 20 years ago. ${ }^{3}$ The contrast was significantly lower than at $1300 \mathrm{~nm}$ and simulated lesions could not be imaged through the full thickness of enamel. ${ }^{3}$ It is also important to point out that stains interfere significantly at $780 \mathrm{~nm}^{15}$ and the contrast between sound and demineralized enamel is markedly higher at wavelengths beyond $1400 \mathrm{~nm}$ in reflectance measurements. ${ }^{15-17}$

Epidemiological data gathered from the National Health and Nutritional Survey ${ }^{18,19}$ and Dental Practice-Based Research Network ${ }^{20-22}$ indicates that nearly one third of all patients have a "questionable" occlusal carious lesion (QOC) located on a posterior tooth. QOCs are given the name questionable because clinicians' lack instrumentation capable of measuring the depth of pit and fissure lesions and determining if the dental decay has reached the underlying dentin. Bitewing radiographs are not sensitive enough to detect most occlusal lesions, and visible diagnosis is confounded by stain trapped in the occlusal anatomy. ${ }^{15}$ Prior in vitro studies attempted to combine SWIR reflectance and transillumination measurements to estimate QOC depth and severity. ${ }^{7,23,24}$ Salsone et al. ${ }^{25}$ and Zakian et al. ${ }^{26}$ used multiple wavelengths of SWIR hyperspectral reflectance images to estimate the severity of occlusal lesions. Since, multispectral SWIR reflectance, and transillumination experiments have demonstrated that the tooth appears darker at wavelengths coincident with increased water absorption, multispectral images can be used to produce increased contrast between different tooth structures such as sound enamel and dentin, dental decay, and composite restorative materials. ${ }^{26-28}$ Combining measurements from different SWIR imaging wavelengths and comparing them with concurrent measurements acquired by complementary imaging modalities should provide improved assessment of lesion depth and severity.

Both the reflectance and the occlusal transillumination probes sample light that is emitted from tooth occlusal surfaces, therefore it is feasible to combine both methods into a single probe that can be positioned above the tooth for rapid clinical screening. Different illumination wavelengths can be used that are optimized for each imaging mode, namely, SWIR wavelengths $>1400 \mathrm{~nm}$ for reflectance and $1300 \mathrm{~nm}$ for transillumination. Simon et al. ${ }^{29}$ built a benchtop simultaneous SWIR reflectance and transillumination system with tunable filters that ranged from 830 to $1700 \mathrm{~nm}$ and showed that the combined images have potential for the diagnosis of QOCs. In a study, the following year using the same benchtop system and simulated approximal lesions showed that multispectral combined images can potentially be used to improve the differentiation of cavitated and noncavitated approximal lesions. ${ }^{30}$ In a third study carried out in 2018, Simon et al. ${ }^{24}$ used the same benchtop system to image 37 extracted teeth with occlusal caries lesions and sectioned the teeth and measured the lesion depth with PLM. This 2018 study showed that the contrast of reflectance at 1500 to $1700 \mathrm{~nm}$ correlated with the lesion depth while the transillumination at 1200 to $1700 \mathrm{~nm}$ showed no correlation. The lesion width, the lesions' buccal-lingual dimension along the fissure, showed a positive correlation with lesion depth for reflectance and transillumination. These initial investigations demonstrated the potential of acquiring simultaneous multiwavelength SWIR reflection and transillumination images to 
improve the detection of caries lesions on both occlusal and proximal surfaces. We hypothesize that the greatest utility of a combined SWIR reflectance and transillumination clinical probe will be to reduce false positives (FPs) since it is unlikely that confounding structural features or specular reflection are going to be present in both reflectance and transillumination images. In addition, the dual probe will provide complementary diagnostic information about lesion severity to help discriminate early superficial lesions on tooth surfaces from deeply penetrating lesions.

In this study, a system for the acquisition of simultaneous SWIR reflectance and transillumination system suitable for clinical use was developed and tested on 120 extracted teeth with occlusal and approximal lesions using micro-computed tomography $(\mu \mathrm{CT})$ as a gold standard.

\section{Materials and Methods}

\subsection{Sample Preparation}

Teeth with no identifiers were collected from patients in the San Francisco Bay area and Geneva Switzerland with approval from the UCSF Committee on Human Research. Extracted teeth $(n=120)$ were selected with occlusal and approximal lesions for this study. Teeth were sterilized using gamma radiation and stored in $0.1 \%$ thymol solution to maintain tissue hydration and prevent bacterial growth. Then, samples were mounted in black orthodontic acrylic blocks from Great Lakes Orthodontics (Tonawanda, New York) and imaged with digital radiographs using a CareStream 2200 System from Kodak (Rochester, New York) operating at $60 \mathrm{kV}$.

All teeth were imaged using $\mu \mathrm{CT}$ with a $10-\mu \mathrm{m}$ resolution. A Scanco $\mu \mathrm{CT} 50$ from Scanco USA (Wayne, Pennsylvania) located at the UCSF Bone Imaging Core Facility was used to acquire the images. Visible color images of the samples were acquired using a USB microscope, Model AM7915MZT from AnMO Electronics Corp. (New Taipei City, Taiwan) with extended depth of field and cross polarization. The digital microscope captures 5 mega-pixel $(2952 \times 1944)$ color images.

\subsection{Design and Fabrication of the Dual SWIR probe}

The dual probe was designed in Fusion 360 from Autodesk (San Francisco, California). The dual probe design consists of a handpiece with reflectance and a transillumination attachment, shown in Figs. 1(a)-1(c). The handpiece incorporated an insert [Fig. 1(d)] to attach the optical fiber for reflectance along with a 5-mm Thorlabs (Newton, New Jersey) polarizing beam splitter cube. The insert utilized black resin to reduce artifacts from unwanted scattering in the handpiece. A cross-sectional view of the reflectance probe body is shown in Fig. 1(c). Light from a fiber optic cable travels from the back of the handpiece to the insert [Fig. 1(d)] and is directed at a right angle by the polarizing beam splitter cube (6) toward the tooth surface as shown by the path of the red arrow in Fig. 1(c). Reflected and transmitted light from the tooth is reflected off a polished aluminum surface (7) attached at a 45-deg angle at the distal end of the handpiece. There is an air nozzle positioned on the bottom of the probe (3) directed toward the aluminum reflector to prevent fogging of the aluminum surface. The air nozzle can also be used to dry the tooth surface to increase lesion contrast and potentially assess lesion activity. ${ }^{31-33}$

The transillumination attachment [Fig. 1(e)] attaches to the distal end of the handpiece as shown in Fig. 1(b). It was fabricated with Formlabs (Boston, Massachusetts) Flexible Resin. The flexible resin can tolerate moderate stretch and compression, making it an ideal choice for imaging teeth with different shapes and sizes. The transillumination optical fibers enter each arm of the attachment and are inserted into the two ports at the rear of the appliance (8) as shown in Figs. 1(b) and 1(e) and into Teflon plugs (9). The Teflon plugs diffusely spread the light from the optical fiber into the tooth on the buccal and lingual surfaces below the dentinal enamel junction and into the gingival tissues when used clinically. They were fabricated from $1 / 8^{\prime \prime}$ diameter Teflon rod cut to a length of $3 \mathrm{~mm}$. The separation of each arm is $1 \mathrm{~cm}$ and they are flexible and can stretch to almost $2 \mathrm{~cm}$ to accommodate larger teeth. 

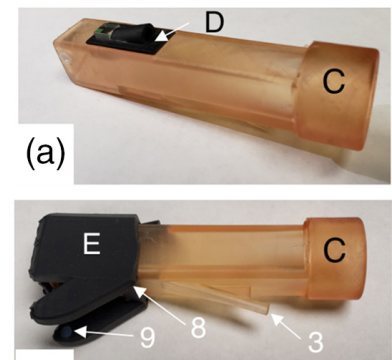

(b)

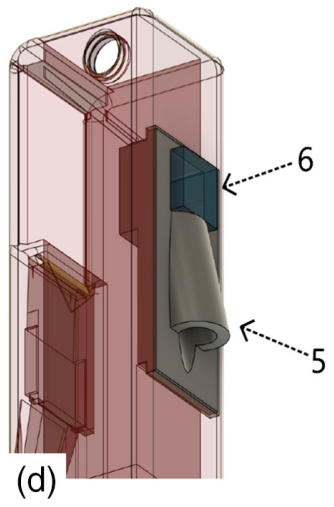

(c)

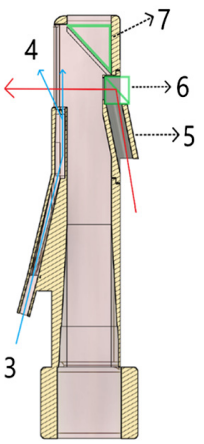

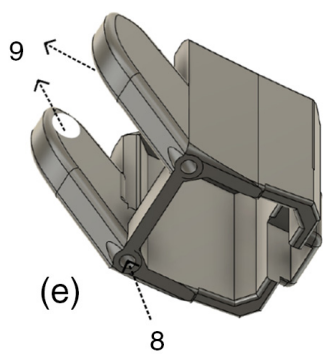

Fig. 1 (a) 3D printed handpiece for reflectance with insert (d) made of black resin with a polarizing beamsplitter cube (6); (b) Handpiece with attachment for transillumination (e). (c) Handpiece cross-sectional view; the reflectance fiber is inserted through the port at (5) from the back of the polarization chamber, and light is polarized (6) and delivered to the tooth occlusal surface as shown by the red arrow. Air enters the nozzle at (3) and is directed toward the tooth surface at (4) as shown by the blue arrows. Reflected and transmitted light from the tooth is directed to the camera by a right angle polished surface (7). The insert for the polarizing beamsplitter cube (d) is snapped to the back of the handpiece. Transillumination fibers are inserted into the two ports (8) at the rear of the transillumination appliance (e) and into the Teflon plugs (9) at the end of each probe arm. The transillumination appliance (e) is placed over the end of the reflectance attachment as shown in (b) and Fig. 2.

The handpiece was fabricated using a Formlabs Form 3 Low Force Stereolithography 3D printer. The final design is exported as a Standard Tessellation Language (STL) file and transferred to Formlabs PreForm to generate supports for a final 3D printing scheme. A spatial resolution of $100 \mu \mathrm{m}$ was used for all prints. Formlabs Dental SG Resin was used for printing the reflectance probe to provide biocompatibility and autoclavability. After 3D printing, handpiece was transferred to Formlabs Form Wash to rinse off the resin residue with isopropyl alcohol for $5 \mathrm{~min}$ and cure for $30 \mathrm{~min}$ at $60^{\circ} \mathrm{C}$ in the Form Cure. The attachment [Fig. 1(d)] housing the 5-mm polarization beam splitting (PBS) cube was printed using Formlabs black resin. After curing, supports are removed with a clip and surfaces are trimmed with water sanding technique to remove sharp edges and achieve smooth finishing.

To assemble the dual SWIR probe, the insert with the PBS cube [Fig. 1(d)] is attached to the top of the handpiece. The aluminum reflector (7) is attached to the distal end of the handpiece and held in place with a set screw. The transillumination attachment [Fig. 1(e)] slides on top of the handpiece and securely locks the insert with the beamsplitter cube (6) in place.

\subsection{Image Acquisition and Analysis}

The SWIR reflectance images were captured using a Model GA1280J (Sensors Unlimited, Princeton, New Jersey) camera with a $1280 \times 1024$ pixel format, a $15-\mu \mathrm{m}$ pixel pitch, and a bit depth of 12-bit. Two 1-in. diameter planoconvex antireflection coated lenses of 60- and 100-mm focal length along with an adjustable aperture were placed between the handpiece and the InGaAs camera to provide a field of view of $11 \times 11 \mathrm{~mm}^{2}$ at the focus plane. A low-OH 


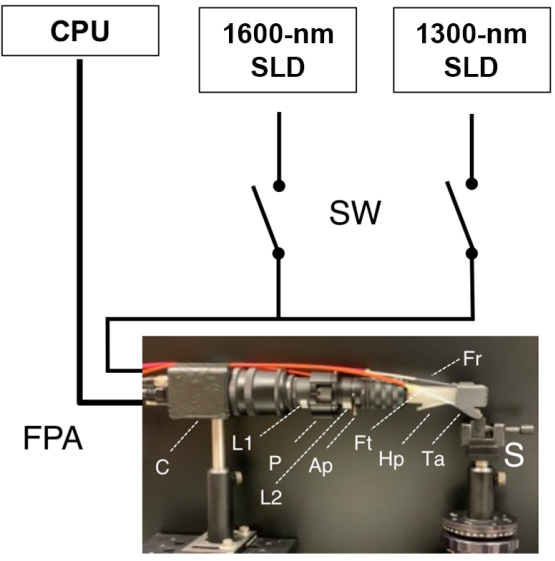

(a)

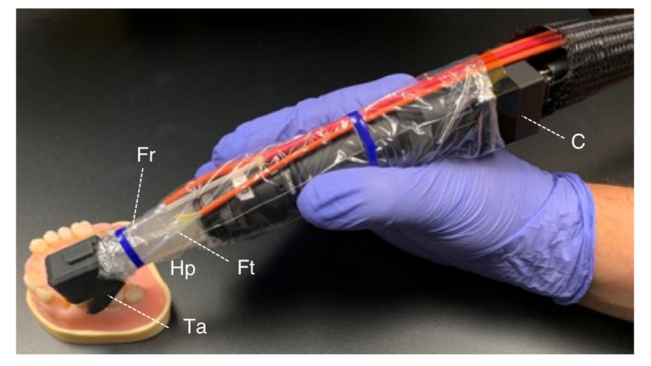

(b)

Fig. 2 (a) Diagram of dual imaging system which employs a computer, two SLDs, two fiber optic switches (SW), and an InGaAs FPA with external optics and handpiece which is position over a sample tooth (S). The camera (C), 100-mm lens (L1), polarizer (P), 60-mm lens (L2), adjustable aperture (Ap), reflectance handpiece $(\mathrm{Hp})$, and transillumination attachment $(\mathrm{Ta})$ are shown along with the orange optical fibers for reflectance $(\mathrm{Fr})$ and transillumination $(\mathrm{Ft})$. (b) The handheld dual imaging handpiece equipped with the smaller InGaAs camera that will be used for clinical imaging.

optical fiber of 1-mm diameter was used to deliver light from a 1604-nm superluminescent diode (SLD), Model ESL 1620-2111 from Exalos (Schlieren, Switzerland) with an output of $17 \mathrm{~mW}$ and a bandwidth of $46 \mathrm{~nm}$. The intensity delivered to the tooth was $5 \mathrm{~mW}$. The transillumination light is delivered through two 0.4-mm diameter low-OH optical fibers. A 1314-nm SLD, Model DL-CS3452A-FP 1620-2111 from Denselight (Singapore) with an output of $48 \mathrm{~mW}$ and a bandwidth of $33 \mathrm{~nm}$ was used as the source for transillumination. A 50/50 beamsplitter was used to deliver light to each arm for transillumination. The output intensity of each arm was set at $10 \mathrm{mw}$ before entering the teflon plugs. The reflectance and transillumination light sources output were controlled via two OSW12(22) MEMS fiber optic switches from Thorlabs. The camera and the optic switch were controlled in custom authored programs in LabView (Austin, Texas). The two optical switches alternate between the 1300-nm light source for transillumination and the 1600$\mathrm{nm}$ source for reflectance allowing near-simultaneous acquisition at a rate of $30 \mathrm{~Hz}$. A diagram of the setup along with an image of the with imaging optics and the assembled dual probe is shown in Fig. 2(a). Figure 2(a) shows the larger Model GA1280J InGaAs camera that was used to acquire the in vitro images used in this study. The probe can also be used with the smaller $640 \times 480$ pixel SWIR camera (SU640CSX) measuring only $32 \times 32 \times 28 \mathrm{~mm}^{3}$ from Sensors Unlimited (Princeton, New Jersey) that is better suited for clinical imaging and it is shown in Fig. 2(b) with the assembled probe.

The samples were dried of excess water with an air nozzle before imaging due to the strong water absorption at $1600 \mathrm{~nm} .{ }^{4}$ Image processing of the images was performed by custom scripts written using MATLAB from Mathworks (Natick, Massachusetts). The acquired 12-bit images (4096) were converted to 16-bit (65536) by multiplying by 16 and subtracting 1 to facilitate processing using MATLAB. Lesion regions of interest (ROIs) were identified from the 
transillumination/reflectance images. Co-registration was unnecessary since they were acquired near simultaneously by the same imaging optics. Sound areas for comparison were chosen from areas surrounding the lesion. This better represents the contrast between the lesion and sound tissues that would be viewed by the clinician. The contrast was calculated for each lesion using the formula $\left(I_{L}-I_{S}\right) / I_{L}$ for reflectance images and $\left(I_{S}-I_{L}\right) / I_{S}$ for transillumination images, where $I_{L}$ is the average intensity in the lesion area ROI, and $I_{S}$ is the average intensity in the sound ROI. ${ }^{5}$ Lesion areas were confirmed using the $\mu \mathrm{CT}$ images.

In addition to calculating the lesion contrast in transillumination and reflectance images, a dual fusion mode combining the two images was created in MATLAB and included in the analysis utilizing the weight, or fraction of the intensity applied from each image. The dual fusion mode is a weighted linear combination of the intensities of the inverted transillumination $\left(I_{\text {Tinv }}=65535-I_{T}\right)$ and the reflectance $\left(I_{R}\right)$ 16-bit images. The lesion contrast in fusion mode was calculated using $\alpha I_{\text {Tinv }}+b I_{R}$, with alpha $(\alpha)$ ranging from 0 to 1 , where $\alpha=1-b$.

\subsection{Scoring of Digital Radiographs and SWIR Images by Clinicians}

Three clinical examiners participated in the study, two examiners had several years of research experience with SWIR imaging and one examiner had no prior experience with SWIR imaging. A training set was used to standardize the knowledge of the three examiners prior to the study. All examiners were presented with radiographs and SWIR images of 120 teeth and asked to score the occlusal, mesial approximal, and distal approximal surfaces of each tooth. At least one of the examiners indicated that at least one of the images for 14 of the teeth was unreadable. Therefore 106 sets of images with four different imaging methods (transillumination, reflectance, dual, and both) were evaluated. The order of the images was randomized for each of the four testing sets among the three examiners. The examiners were asked to provide a binary score (lesion or no lesion) for each of the three surfaces. Diagnostic accuracy was calculated using $(\mathrm{TP}+\mathrm{TN}) /(\mathrm{TP}+\mathrm{TN}+\mathrm{FP}+\mathrm{FN})$ where $\mathrm{TP}, \mathrm{TN}, \mathrm{FP}$, and $\mathrm{FN}$ are true positives, true negatives, false positives and false negative, respectively. $\mu \mathrm{CT}$ was used as the gold standard. The percent agreement for each tooth was calculated, and then the average $\%$ agreement was calculated for all the samples to represent the study interrater reliability. Clinician scores of interrater reliability (percent agreement), accuracy, and FP were calculated for the four SWIR image types and the radiographs, and the values are tabulated in Table 2.

\section{Results}

Several images from a tooth with an occlusal lesion in a fissure that penetrates almost to the underlying dentin are shown in Fig. 3. The visible image of the occlusal surface [Fig. 3(a)] shows a mandibular premolar with red/brown staining in the central fissure and mesial pit, however, there is no evidence of increased white (value) areas that are indicative of visible demineralization on the tooth surface. The SWIR reflectance image [Fig. 3(b)] shows with high contrast increased light scattering from the stained fissure and pit indicating that the enamel is demineralized in these areas. There are also some other areas of high intensity, particularly on the buccal cusp tip that may be due to demineralization but is more likely specular reflection from the surface. There is no decay visible in the radiograph [Fig. 3(c)] while the $\mu \mathrm{CT}$ slice clearly shows that the V-shaped lesion in the fissure has penetrated almost through the enamel. The decay in the fissure and the pit is also detectable in the transillumination image [Fig. 3(e)] increasing the probability that those areas are actually demineralized and not due to an optical anomaly. The bright spot originating from the buccal cusp tip in the reflectance image is not visible in the transillumination image and is likely an area of specular reflection. The fusion image [Fig. 3(f)] shows the lesion areas with high contrast, however, it also highlights areas of suspected specular reflection.

Several images of another tooth with approximal lesions at both proximal contact points are shown in Fig. 4. The visible image of the occlusal surface shows nearly no evidence of approximal lesions [Fig. 4(a)] and only one of the lesions is visible in the radiograph [Fig. 4(c)] and it appears to penetrate less than halfway through the enamel. The SWIR reflectance, 


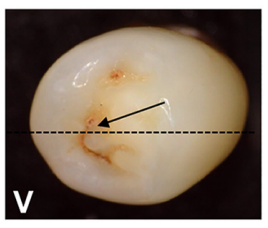

(a)

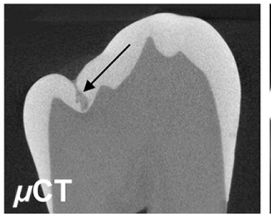

(d)

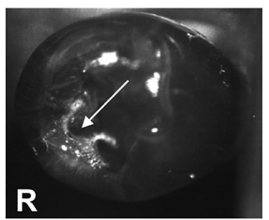

(b)

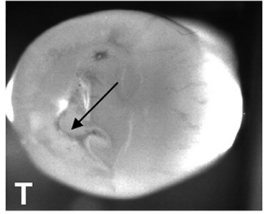

(e)

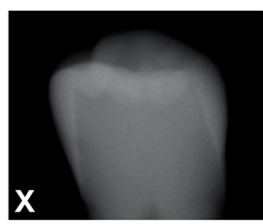

(c)

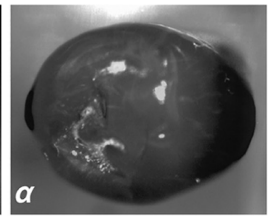

(f)

Fig. 3 (a) Visible; (b) SWIR reflectance at $1600 \mathrm{~nm}$; (c) dental radiographic; (d) $\mu$-CT, (e) SWIR transillumination at $1300 \mathrm{~nm}$; and (f) combined $\mathrm{R}+\mathrm{T}$ fusion $(\alpha=0.4)$ images of a tooth with an occlusal lesion. Arrows point to the occlusal lesion and the dotted line in (a) shows the orientation of the slice in the $\mu \mathrm{CT}$ image.

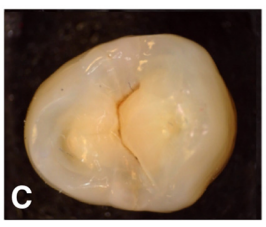

(a)

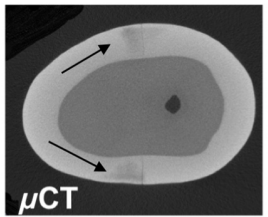

(d)

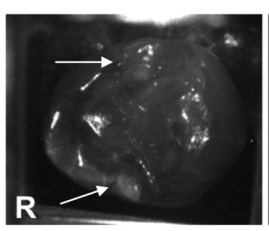

(b)

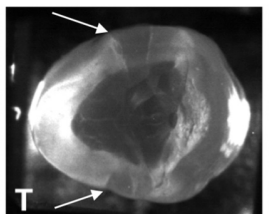

(e)

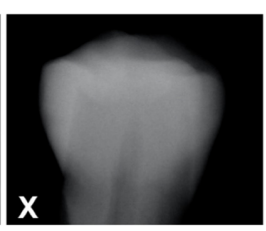

(c)

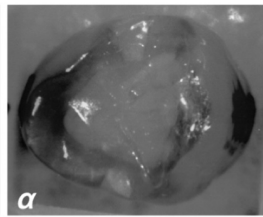

(f)

Fig. 4 (a) Color; (b) SWIR reflectance at $1600 \mathrm{~nm}$; (c) dental radiographic; (d) $\mu-\mathrm{CT}$; (e) SWIR transillumination at $1300 \mathrm{~nm}$; and (f) combined $\mathrm{R}+\mathrm{T}$ fusion $(\alpha=0.4)$ images of a tooth with two approximal lesions. Arrows point to lesions.

transillumination, and $\mu \mathrm{CT}$ images agree quite well all showing that both lesions penetrate almost to the dentinal enamel junction (DEJ). The fusion image [Fig. 4(f)] shows the lesions with high contrast and the surrounding sound enamel areas appear more uniform in intensity.

Figure 5 shows the effect of varying alpha $\left(\alpha I_{\text {Tinv }}+b I_{R}\right)$ in the dual fusion images. Images with $\alpha$ values varying from 0.0 to 0.9 are shown of a tooth with an approximal lesion. For this particular tooth, the contrast of the lesion increased with increasing $\alpha$. The contrast did not increase with increasing $\alpha$ for all of the teeth, some exhibited a decrease with increasing $\alpha$. The mean lesion contrast from all samples is plotted as a function of the $\alpha$ coefficient in Fig. 6 for occlusal lesions (circles, $n=120$ ) and approximal lesions (triangles, $n=120$ ). The approximal lesion depth was measured from the proximal surface contact point to deepest lesion point in the pulpal direction while the lesion depth for occlusal lesions was measured from the occlusal surface to deepest lesion point in the pulpal direction.

The mean lesion contrast is plotted versus lesion depth for occlusal lesions in Fig. 7 for reflectance, transillumination, and the fusion mode images. The reflectance and transillumination measurements were all from the occlusal surface. There was no significant dependence on the lesion contrast with lesion depth. For approximal lesions, the lesion contrast was determined versus the distance of the lesion from the occlusal surface. This comparison is shown in Fig. 8 for 

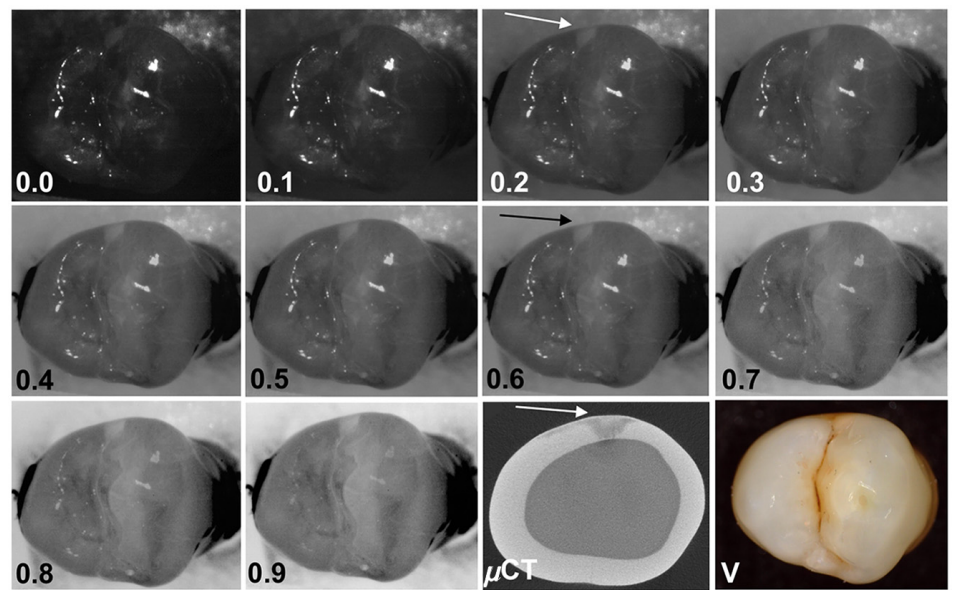

Fig. 5 Combined transillumination and reflectance images with a varying from 0 to 0.9 along with visible (V) and an occlusal ( $\mu \mathrm{CT}) \mu \mathrm{CT}$ image of a tooth with occlusal decay. For this particular tooth the contrast increased with increasing $a$.

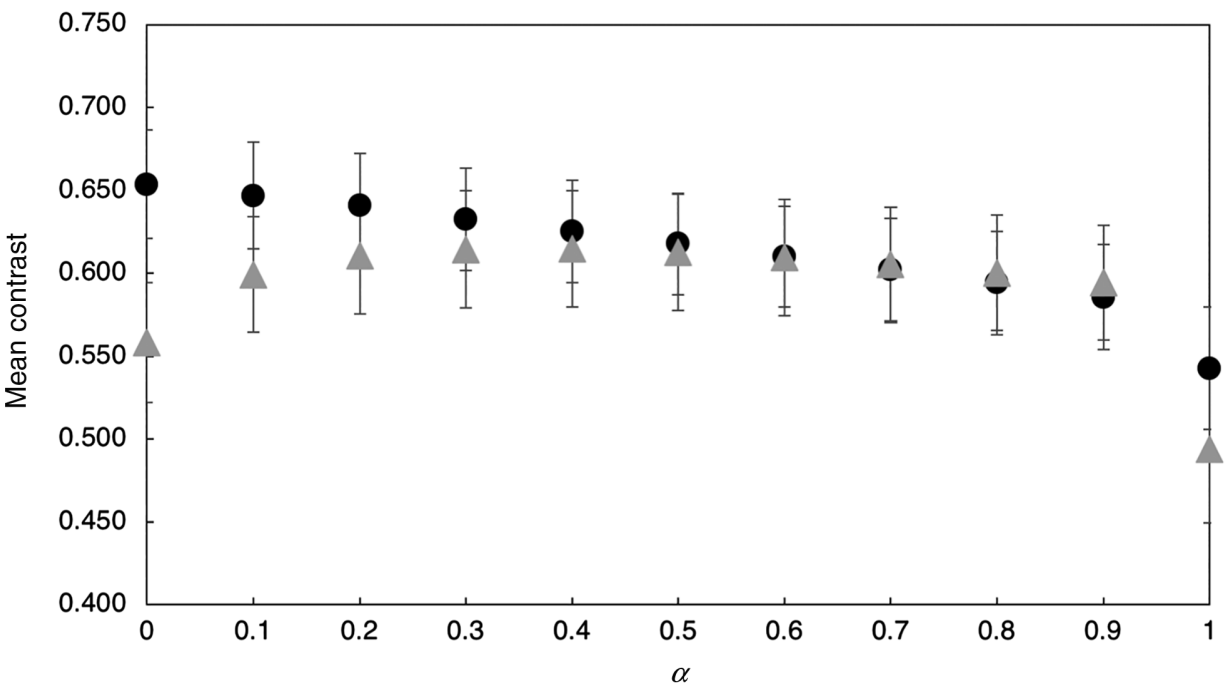

Fig. 6 Plot of mean \pm SD of lesion contrast versus $\alpha$ for dual-probe fusion mode $(\alpha=0.4)$ images for occlusal lesions (circles, $n=120$ ) and approximal lesions (triangles, $n=120$ ).

the reflectance and transillumination images along with the fusion images and there was also no significant dependence on the lesion contrast with distance from the occlusal surface.

The effect of $\alpha$ on the mean lesion contrast is tabulated in Table 1 for occlusal and approximal lesions. For occlusal lesions, the reflectance mode alone performs exceptionally as it shows a mean lesion contrast of 0.65 versus 0.57 from transillumination mode. Dual fusion mode negatively impacts the mean occlusal lesion contrast, and as $\alpha$ gets higher the mean lesion contrast decreases. For approximal lesions, $\alpha=0.4$ shows a mean lesion contrast of 0.615 , improving the contrast of $49.4 \%$ of the approximal lesions and showing contrast enhancement of $8.2 \%$ from the reflectance mode and $22.8 \%$ from the transillumination mode.

The examiner agreement (percent agreement), accuracy, and FP rate are given in Table 2 for the three clinician assessments of the SWIR images and the radiographs. The mesial and distal proximal surfaces were combined for the table. The examiner agreement was highest for the radiographs. The examiner agreement was moderate for transillumination, reflectance, merged images, and side by side images for both occlusal and proximal surfaces. Reflectance yielded the highest accuracy and lowest FP rate while transillumination yielded the lowest accuracy and 
Zhu et al.: Dual short wavelength infrared transillumination/reflectance mode imaging...

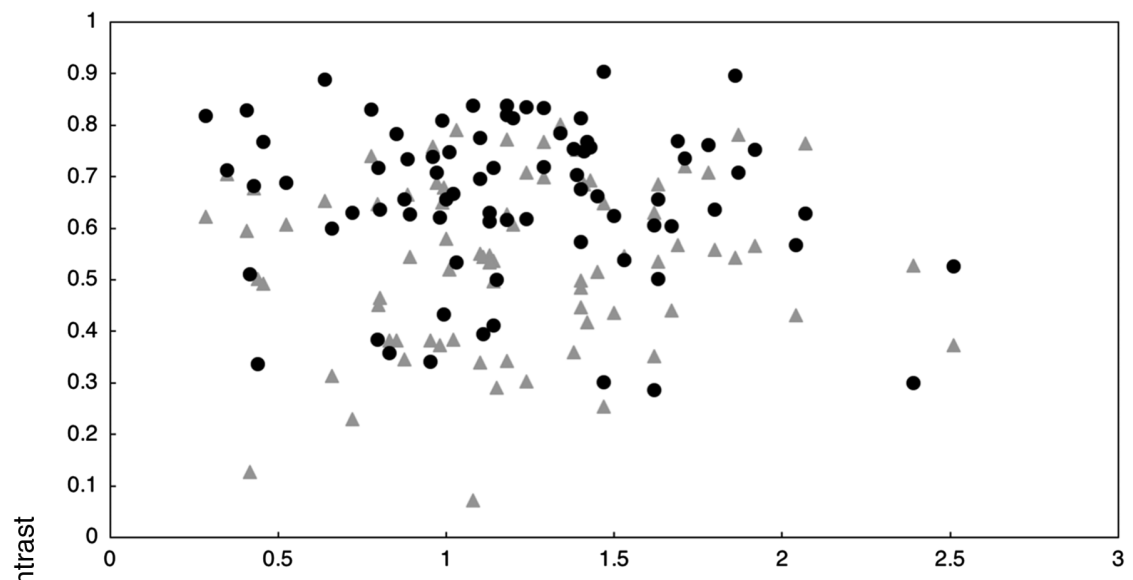

(a)

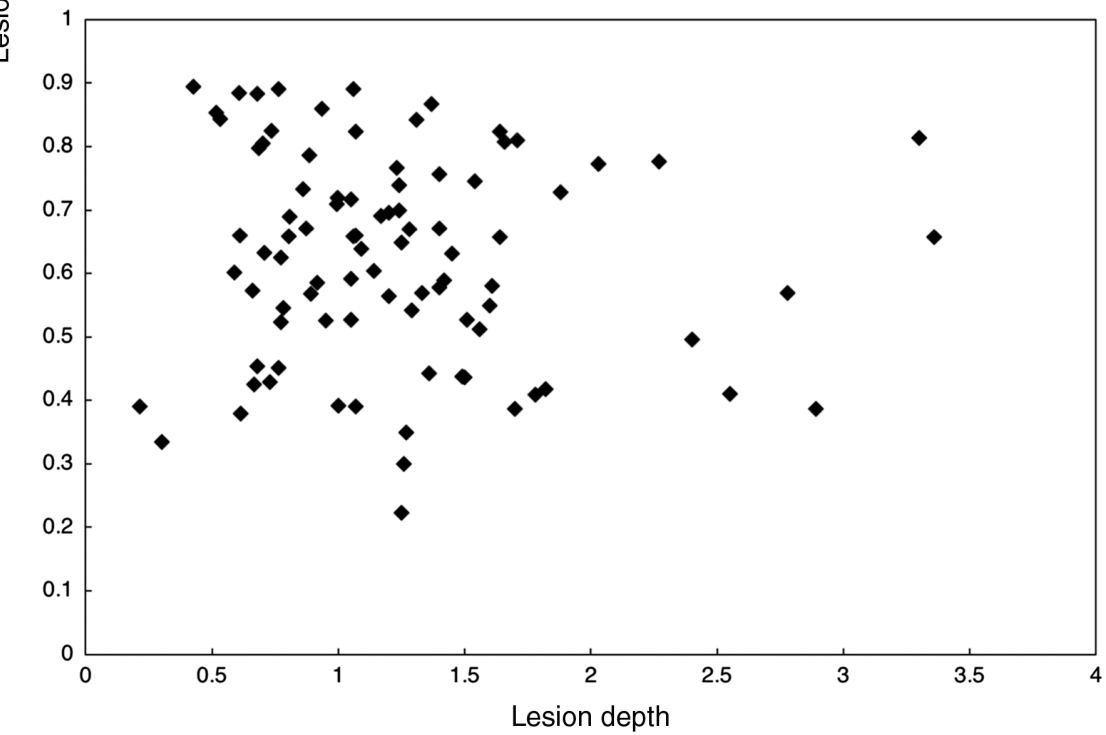

(b)

Fig. 7 Occlusal lesion contrast versus lesion depth measured with $\mu \mathrm{CT}$. (a) Reflectance (circles) and transillumination (triangles). (b) fusion mode images $(\alpha=0.4)$.

the highest FP rate among SWIR imaging. The use of dual mode yielded a slight improvement in accuracy, percent agreement, and FP rate over transillumination alone but was inferior to reflectance. The use of side-by-side reflectance and transillumination images did not appear to improve diagnostic performance or reduce the FP rate. Both the FP rate and the accuracy of radiographs were lower than the SWIR methods.

\section{Discussion}

In this study, a compact dual reflectance and transillumination SWIR imaging device was employed to acquire images of 120 extracted teeth with caries lesions. Near-simultaneous reflectance and occlusal transillumination images are collected from the tooth occlusal surface which facilitates registration and integration. The reflectance probe delivers 1600-nm light to the tooth occlusal surface while the occlusal transillumination probe delivers 1300 -nm light cervically at the lingual and buccal sides of the tooth allowing it to propagate into the tooth diffuse in the cervical-coronal direction interacting with approximal and occlusal lesions and exiting the occlusal surface. The transillumination probe utilizes light at $1300 \mathrm{~nm}$ where the enamel is most transparent. Scattering in enamel continues to decrease with increasing wavelength beyond 
Zhu et al.: Dual short wavelength infrared transillumination/reflectance mode imaging...

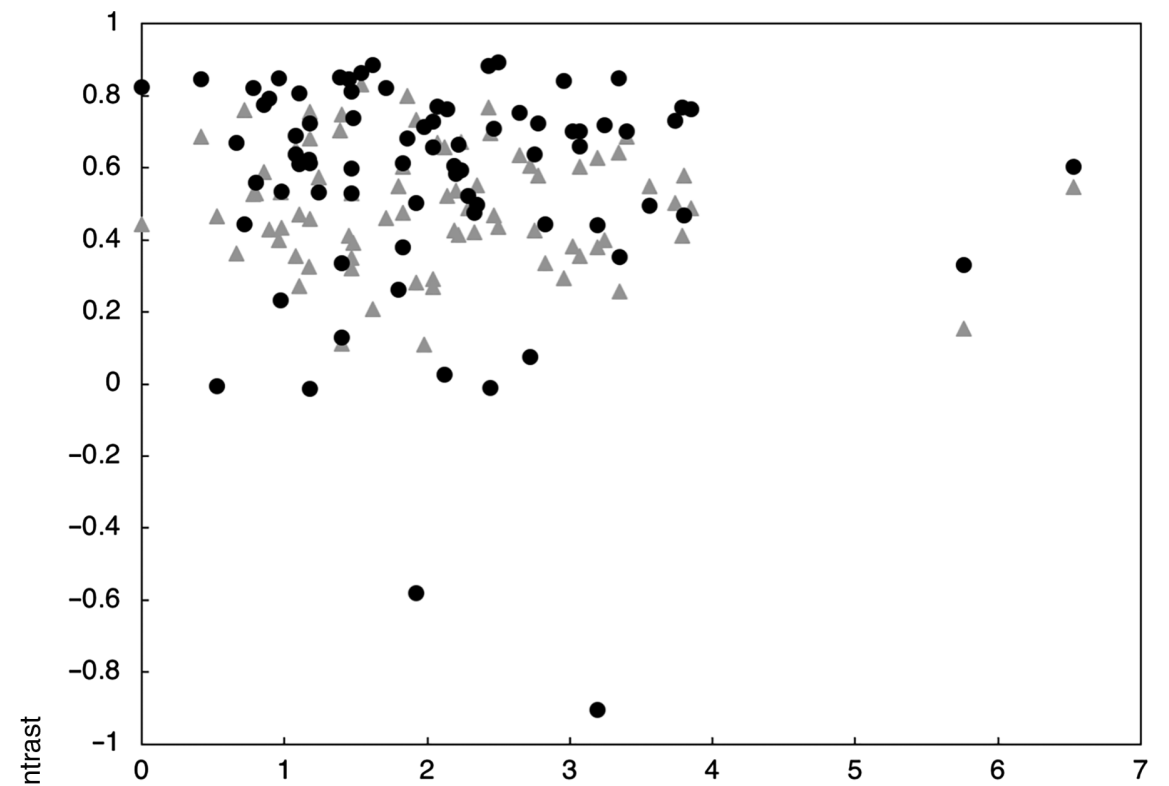

(a)

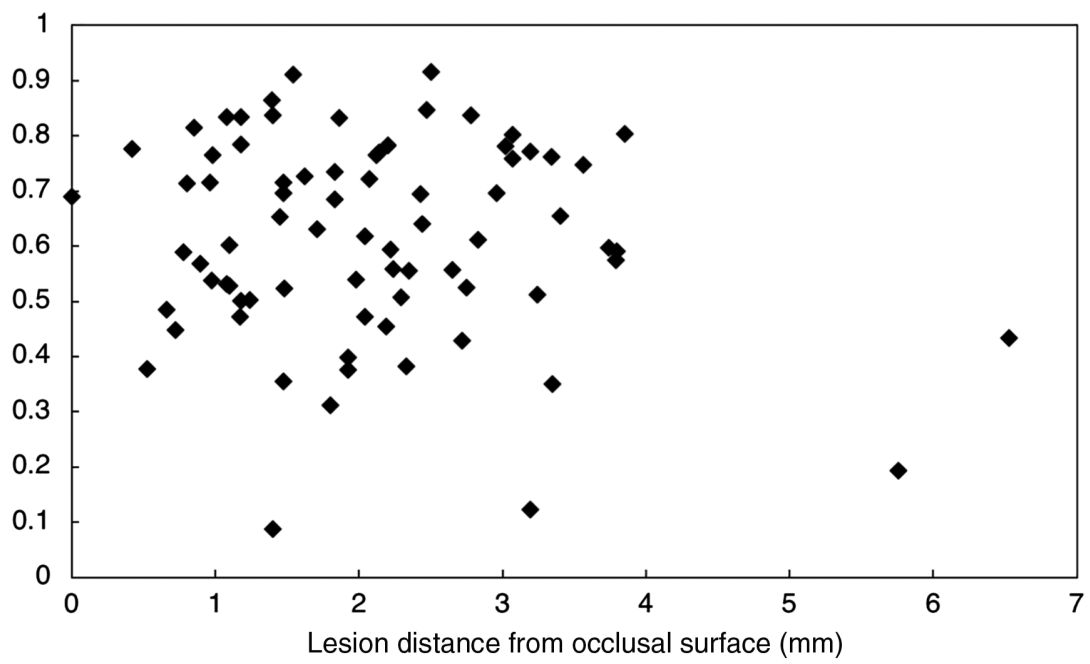

(b)

Fig. 8 Approximal lesion contrast versus lesion distance from the occlusal surface measured with $\mu$ CT. (a) Reflectance (circles) and transillumination (triangles). (b) fusion mode $(\alpha=0.4)$ images.

Table 1 Effect of $\alpha$ on the mean lesion contrast for occlusal and approximal lesions.

\begin{tabular}{|c|c|c|c|c|c|c|c|c|c|c|c|}
\hline$\alpha$ & $(\alpha=0) \mathrm{R}$ & 0.1 & 0.2 & 0.3 & 0.4 & 0.5 & 0.6 & 0.7 & 0.8 & 0.9 & $(\alpha=1)(\mathrm{T})$ \\
\hline Occlusal lesion contrast & 0.651 & 0.641 & 0.631 & 0.621 & 0.612 & 0.602 & 0.594 & 0.584 & 0.575 & 0.566 & 0.533 \\
\hline Approximal lesion contrast & 0.558 & 0.599 & 0.611 & 0.614 & 0.615 & 0.613 & 0.610 & 0.605 & 0.6 & 0.595 & 0.494 \\
\hline
\end{tabular}

$1300 \mathrm{~nm}$, however, water absorption increases markedly beyond $1400 \mathrm{~nm}$ greatly increasing attenuation. ${ }^{34}$ The dual SWIR probe can capture high definition in-vitro transillumination and reflectance SWIR images of extracted teeth. The probe was fabricated using a 3D printer and utilized two broadband fiber-optic SLD light sources. Previous studies have investigated the contrast of caries lesions using both reflectance and transmitted light. This was the first study demonstrating the performance of an imaging system that simultaneously acquires 
Table 2 Clinician scores for the occlusal and proximal surfaces of 106 teeth for percent agreement, accuracy, and FP rate for SWIR imaging methods and radiography.

\begin{tabular}{llcc}
\hline \hline Examiners & \multicolumn{1}{c}{ Method } & Occlusal & Proximal \\
\hline Percent agreement & Transillumination & 0.75 & 0.76 \\
& Reflectance & 0.87 & 0.84 \\
& Dual & 0.80 & 0.79 \\
& Side by side & 0.82 & 0.80 \\
Radiography & 0.88 & 0.91 \\
Tccuracy & Transillumination & 0.45 \\
& Reflectance & 0.54 & 0.49 \\
& Dual & 0.81 & 0.53 \\
FP rate & Side by side & 0.71 & 0.55 \\
& Radiography & 0.70 & 0.49 \\
& Transillumination & 0.36 & 0.55 \\
Reflectance & 0.50 & 0.36 \\
Dual & 0.31 & 0.39 \\
Side by side & 0.47 & 0.36 \\
& Radiography & 0.36 & 0.16 \\
\hline
\end{tabular}

cross-polarized reflectance and occlusal transillumination images at multiple SWIR wavelengths using a single compact handpiece suitable for clinical use. In addition, it was the first study to compare the SWIR lesion contrast with $\mu \mathrm{CT}$ images as the gold standard for lesion depth.

The primary motivation for acquiring dual-mode images is to reduce the probability of FPs and remove the need to switch between transillumination and reflectance probes during screening. In SWIR occlusal transillumination, cracks and structural features in the tooth can block light propagation through the tooth producing features that resemble lesions, greatly increasing the potential for FPs. Specular reflection interferes with SWIR reflectance measurements and even when high extinction ratio polarizers are employed, there is still substantial specular reflection present in cross-polarization images that have the potential to generate additional FPs. Even though studies are lacking indicating that NIR imaging leads to an increased number of FPs, clinicians using this new technology have voiced concern regarding FPs. Moreover, the markedly higher sensitivity of NIR and SWIR imaging compared to radiography is likely to lead to an increase in FPs simply on the basis of the higher sensitivity. In our most recent clinical study involving SWIR imaging of teeth scheduled to be extracted with unknown caries status, the specificity of SWIR imaging was lower than for radiography, although the difference was not statistically significant. ${ }^{9}$ In that study images of the teeth were acquired using three separate imaging probes: SWIR reflectance $(1600 \mathrm{~nm})$, SWIR occlusal transillumination $(1300 \mathrm{~nm})$, and SWIR proximal transillumination $(1300 \mathrm{~nm})$, combining multiple images for diagnostic performance improved both the sensitivity and the specificity.

Another important advantage of imaging in the SWIR beyond $1150 \mathrm{~nm}$ is that the chromophores responsible for stains on teeth do not absorb light at longer wavelengths since there is not sufficient energy for electronic excitation. ${ }^{1,26}$ This is of particular importance in the stained pits and fissures of tooth occlusal surface. $\mathrm{Ng}$ et al ${ }^{15}$ demonstrated that it is necessary to use SWIR wavelengths $>1150 \mathrm{~nm}$ to avoid significant interference from stains when measuring lesion contrast in reflectance and transillumination modalities. Therefore, stains can be easily differentiated from actual demineralization in the SWIR range, which is not possible at visible 
wavelengths or at NIR/SWIR wavelengths of less than $1150 \mathrm{~nm}$. Chung et al. ${ }^{16}$ showed that absorption due to stains contributed more to the lesion contrast than increased scattering due to demineralization at visible wavelengths and that is clearly demonstrated in Fig. 3. Since it is impractical and unnecessary to remove stains from the deep grooves and fissures on tooth occlusal surfaces, lack of interference from stains at longer SWIR wavelengths is a significant advantage.

Dental radiographs suffer from very low contrast, and it takes considerable experience and expertise to accurately read such images. The principal advantage of radiographs is the very high specificity, i.e., if a lesion is visible in a radiograph it is most likely a TP, therefore, overtreatment is less likely. In addition to the low sensitivity, radiographs typically underestimate the penetration depth of approximal lesions and dentists typically have to estimate that the lesions penetrate much deeper than they appear based on subjective factors involving caries risk status. Images acquired in this study suggest that SWIR images better represent the true depth of the approximal lesions and better match the depth indicated in $\mu \mathrm{CT}$ images than radiographs. SWIR images are also quantifiable and provide measurements that can be utilized for non-biased assessment of lesion depth and severity.

Both reflectance and occlusal transillumination imaging modes yield high contrast for approximal lesions. Since more light propagates through the transparent outer enamel than through the highly scattering dentin core, cracks, fractures, and other defects in the tooth can markedly influence light propagation in the tooth and can produce FPs. For example, two small fractures a few millimeters apart can produce a dark area on the outer ring of enamel that closely resembles an approximal lesion and this is the most common location for cracks in posterior teeth. The same cracks do not interfere with reflectance imaging since the light paths are directly above the lesion. Therefore, access to both reflectance and occlusal transillumination has the potential to increase the sensitivity and specificity of caries detection by optically sampling the entire external surface of existing lesions.

Reflectance imaging is more sensitive than occlusal transillumination for the detection of occlusal lesions. Even shallow demineralization confined to the outer half of the enamel can appear with high contrast in reflectance images. The challenge with SWIR imaging of occlusal lesions is differentiating the very shallow and superficial lesions from the lesions that penetrate through the enamel and into the underlying dentin. It is these deeper penetrating lesions that require removal and restoration. Remineralization therapy is a more appropriate treatment approach for shallow lesions. Previous studies have shown that the contrast increases significantly with increasing depth for SWIR reflectance imaging, however, the magnitude of the change is not sufficient to warrant the use of contrast thresholds to define lesion severity. ${ }^{35}$ Multiple studies including this one show that there is no dependence of the lesion contrast on depth for occlusal transillumination. A more reliable indicator of lesion depth and severity is the lesion size or width in the dimension perpendicular to the fissure or radial to a pit. ${ }^{16,35}$ Severe occlusal lesions typically arise in the plaque collection sites in the pits and fissures and grow from the base of the fissure to the underlying dentin. Upon reaching the dentin the lesion can spread more rapidly laterally along the dentinal-enamel junction. That lateral spread can be easily seen through the transparent sound enamel above the lesion, particularly at SWIR wavelengths and this approach of detecting these hidden occlusal lesions has been demonstrated in vivo using SWIR imaging and optical coherence tomography in two clinical studies. ${ }^{36,37}$ If the lesion becomes severe enough it can become visible to the naked eye as a dark shadow. In this study, there was no significant correlation of the lesion depth and the lesion contrast for either occlusal or approximal lesions for SWIR reflectance or transillumination. This contrasts with our earlier study utilizing SWIR reflectance ${ }^{35}$ where a significant correlation was observed between the lesion contrast and lesion depth for occlusal lesions. That prior study used optical coherence tomography to identify the occlusal lesions while this study used $\mu \mathrm{CT}$, therefore, many of the lesions in the prior study were much shallower than the lesions identified in this study. This is one disadvantage of using $\mu \mathrm{CT}$ over optical coherence tomography, $\mu \mathrm{CT}$ is insensitive to the very shallow lesions that are commonly found on occlusal surfaces. The shallowest lesions imaged in this study were all greater than $0.5 \mathrm{~mm}$ in depth. In reflectance, the reflected intensity is expected to saturate above a given thickness/depth where it no longer increases with increasing depth. ${ }^{38}$ The thickness at which saturation occurs depends on the wavelength and the degree of 
demineralization. For greater lesion depths additional metrics are required for extrapolating potential lesion depth.

In addition to measurements of the lesion contrast, the SWIR images and radiographs were scored by three clinicians and $\mu \mathrm{CT}$ was used as a gold standard. Assessments of diagnostic performance should be carried out in vivo with a selection of teeth with a similar caries incidence expected for that population. That was certainly not the case in this study where all the teeth had lesions, however, the objective of this study was to show that the use of near-simultaneous SWIR reflectance and occlusal transillumination images has the potential for removing FPs and increasing diagnostic performance. Another concern with an in vitro study of this nature is that we have observed that in vivo transillumination images are typically better than in vitro images of lesions on extracted teeth due to the better internal hydration of vital teeth. The loss of internal water from extracted teeth markedly increases the scattering of sound dental tissues and reduces the lesion contrast. ${ }^{16}$ In addition, there were no overlapping or adjoining teeth, such teeth are expected to reduce the performance of radiographs and SWIR transillumination for imaging approximal lesions and would not be expected to influence the imaging of occlusal lesions or influence reflectance imaging. SWIR reflectance alone yielded the highest examiner agreement, highest accuracy, and lowest FP rate among the four image types. The SWIR reflectance alone had a higher accuracy than radiographs for approximal lesions (0.59 versus 0.49$)$ and markedly higher accuracy for occlusal lesions (0.81 versus 0.36$)$. The examiner agreement was highest for radiography and lowest for SWIR transillumination. This suggests that more training is required to read the SWIR images particularly the SWIR transillumination images. It was anticipated that either the dual mode or side by side SWIR images would yield the highest performance. However, the diagnostic performance for SWIR transillumination was quite low and that low performance most likely adversely influenced the dual mode and side by side images. It is likely that the performance of SWIR transillumination is most dramatically reduced in in vitro measurements due to the influence of the gingival tissues and the internal hydration of the tooth. The performance of this handpiece shown in Fig. 2(b) will be assessed in vivo over the next year and we will be able to confirm this hypothesis.

\section{Conclusions}

In summary, we have developed a clinical probe capable of acquiring near-simultaneous SWIR reflectance and occlusal transillumination images of approximal and occlusal lesions. The performance of the probe in vitro was assessed by imaging 120 extracted teeth with lesions on the occlusal and proximal surfaces. The next step will be to image in vivo teeth of unknown caries status scheduled for extraction for orthodontic reasons, to assess the diagnostic performance of this dual probe in vivo.

\section{Disclosures}

No conflicts of interest, financial or otherwise, are declared by the authors.

\section{Acknowledgments}

The authors would like to acknowledge the support of the National Institute of Dental and Craniofacial Research/NIH under Grant Nos. R01-DE028295 and F30-DE026052, and the Swiss National Science Foundation (SNSF) under Grant No. P2GEP3-188157. The authors would like to thank Nick Chang, William Fried, and Vincent Yang and for their contribution to this work. This is an expanded version of SPIE proceeding 11217 0J. ${ }^{39}$

\section{Code, Data, and Materials Availability}

There is no code essential for reproducibility or supplementary data available for this paper. 


\section{References}

1. C. Buhler, P. Ngaotheppitak, and D. Fried, "Imaging of occlusal dental caries (decay) with near-IR light at 1310-nm," Opt. Express 13(2), 573-582 (2005).

2. M. Staninec et al., "In vivo near-IR imaging of approximal dental decay at 1,310 nm," Lasers Surg. Med. 42(4), 292-298 (2010).

3. G. Jones, R. S. Jones, and D. Fried, "Transillumination of interproximal caries lesions with 830-nm light," Proc. SPIE 5313, 17-22 (2004).

4. R. S. Jones et al., "Near-IR transillumination at 1310-nm for the imaging of early dental caries," Opt. Express 11(18), 2259-2265 (2003).

5. D. Fried et al., "Early caries imaging and monitoring with near-IR light," Dent. Clin. North Am. - Incipient Hidden Caries 49(4), 771-793 (2005).

6. K. Hirasuna, D. Fried, and C. L. Darling, "Near-IR imaging of developmental defects in dental enamel.," J. Biomed. Opt. 13(4), 044011 (2008).

7. C. Lee et al., "Nondestructive assessment of the severity of occlusal caries lesions with near-infrared imaging at $1310 \mathrm{~nm}, "$ J. Biomed. Opt. 15(4), 047011 (2010).

8. L. Karlsson et al., "Near-infrared transillumination of teeth: measurement of a system performance," J. Biomed. Opt. 15(3), 036001 (2010).

9. J. C. Simon et al., "Near-IR transillumination and reflectance imaging at 1300-nm and 1500-1700-nm for in vivo caries detection," Lasers Surg. Med. 48(9), 828-836 (2016).

10. J. Kuhnisch et al., "In vivo validation of near-infrared light transillumination for interproximal dentin caries detection," Clin. Oral Investig. 20(4), 821-829 (2015).

11. F. Sochtig, R. Hickel, and J. Kuhnisch, "Caries detection and diagnostics with near-infrared light transillumination: clinical experiences," Quintessence Int. 45(6), 531-538 (2014).

12. M. Abdelaziz and I. Krejci, "DIAGNOcam-a near infrared digital imaging transillumination (NIDIT) technology," Int. J. Esthet. Dent. 10(1), 158-65 (2015).

13. M. Abdelaziz, I. Krejci, and D. Fried, "Enhancing the detection of proximal cavities on near infrared transillumination images with Indocyanine Green (ICG) as a contrast medium: In vitro proof of concept studies," J. Dent. 91, 103222 (2019).

14. M. Abdelaziz et al., "Near infrared transillumination compared with radiography to detect and monitor proximal caries: a clinical retrospective study," J. Dent. 70, 40-45 (2018).

15. C. Ng et al., "Near-Infrared imaging of demineralization on the occlusal surfaces of teeth without the interference of stains," J. Biomed. Opt. 24(3), 036002 (2019).

16. S. Chung et al., "Multispectral near-IR reflectance and transillumination imaging of teeth," Biomed. Opt. Express 2(10), 2804-2814 (2011).

17. W. A. Fried et al., "High contrast reflectance imaging of simulated lesions on tooth occlusal surfaces at near-IR wavelengths," Lasers Surg. Med. 45(8), 533-541 (2013).

18. B. A. Dye et al., "Dental caries and tooth loss in adults in the United States, 2011-2012," NCHS Data Brief 197(197) (2015).

19. B. A. Dye et al., "Trends in oral health status, United States, 1988-1994 and 1999-2004," Vital Health Stat. 248, 1-92 (2007).

20. S. K. Makhija et al., "Characteristics, detection methods and treatment of questionable occlusal carious lesions: findings from the National Dental Practice-Based research network," Caries Res. 48(3), 200-207 (2014).

21. S. K. Makhija et al., "Twenty-month follow-up of occlusal caries lesions deemed questionable at baseline: findings from the National Dental Practice-Based Research Network," J. Am. Dent. Assoc. 145(11), 1112-1118 (2014).

22. S. K. Makhija et al., "The prevalence of questionable occlusal caries: findings from the Dental Practice-Based Research Network," J. Am. Dent. Assoc. 143(12), 1343-1350 (2012).

23. C. Lee, C. L. Darling, and D. Fried, "In vitro near-infrared imaging of occlusal dental caries using a germanium enhanced CMOS camera," Proc. SPIE 7549, 75490K (2010).

24. J. C. Simon et al., "Multispectral near-infrared reflectance and transillumination imaging of occlusal carious lesions: variations in lesion contrast with lesion depth," Proc. SPIE 10473, 1047305 (2018).

25. S. Salsone et al., "Histological validation of near-infrared reflectance multispectral imaging technique for caries detection and quantification," J. Biomed. Opt. 17(7), 076009 (2012). 
26. C. Zakian, I. Pretty, and R. Ellwood, "Near-infrared hyperspectral imaging of teeth for dental caries detection,” J. Biomed. Opt. 14(6), 064047 (2009).

27. H. Tom et al., "Near-infrared imaging of demineralization under sealants," J. Biomed. Opt. 19(7), 77003 (2014).

28. J. C. Simon et al., "Near-infrared imaging of secondary caries lesions around composite restorations at wavelengths from 1300-1700-nm," Dent. Mater. 32(4), 587-595 (2016).

29. J. C. Simon et al., "Transillumination and reflectance probes for near-IR imaging of dental caries," Proc. SPIE 8929, 89290D (2014).

30. J. C. Simon, C. L. Darling, and D. Fried, "Assessment of cavitation in artificial approximal dental lesions with near-IR imaging," Proc. SPIE 1004, 1004407 (2017).

31. R. C. Lee, C. L. Darling, and D. Fried, "Assessment of remineralization via measurement of dehydration rates with thermal and near-IR reflectance imaging," J. Dent. 43, 1032-1042 (2015).

32. R. C. Lee, C. L. Darling, and D. Fried, "Activity assessment of root caries lesions with thermal and near-infrared imaging methods," J. Biophotonics 10(3), 433-445 (2016).

33. R. C. Lee et al., "Infrared methods for assessment of the activity of natural enamel caries lesions," IEEE J. Sel. Top. Quantum Electron. 22(3), 6803609 (2014).

34. K. H. Chan and D. Fried, "Multispectral cross-polarization reflectance measurements suggest high contrast of demineralization on tooth surfaces at wavelengths beyond 1300-nm due to reduced light scattering in sound enamel," J. Biomed. Opt. 23(6), 060501 (2018).

35. J. C. Simon et al., "Multispectral near-IR reflectance imaging of simulated early occlusal lesions: variation of lesion contrast with lesion depth and severity," Lasers Surg. Med. 46(3), 203-215 (2014).

36. J. C. Simon et al., "Near-IR and CP-OCT imaging of suspected occlusal caries lesions," Lasers Surg. Med. 49(3), 215-224 (2017).

37. M. Staninec et al., "Nondestructive clinical assessment of occlusal caries lesions using nearIR imaging methods," Lasers Surg. Med. 43(10), 951-959 (2011).

38. M. van Gemert, A. J. Welch, and W. Star, "One-dimensional transport theory," in OpticalThermal Response of Laser-Irradiated Tissue, M. van Gemert and A. J. Welch Theory, Eds., Springer (2011).

39. Y. Zhu et al., "A dual handheld SWIR transillumination/reflectance probe for imaging lesions on tooth occlusal and proximal surfaces," Proc. SPIE 1121, 112170J (2018).

Biographies of the authors are not available. 